\title{
Long-acting injectable antipsychotics: focus on olanzapine pamoate
}

This article was published in the following Dove Press journal:

Neuropsychiatric Disease and Treatment

26 May 2010

Number of times this article has been viewed

\section{JP Lindenmayer}

Department of Psychiatry, New York University School of Medicine, New York NY, USA
Correspondence:JP Lindenmayer Psychopharmacology Research Unit-Nathan Kline institute for Psychiatric Research, Manhattan Psychiatric Center,Wards Island, New York NY 10035, USA Email lindenmayer@nkl.rfmh.org
Abstract: Medication non-adherence in patients with schizophrenia continues to be a significant problem and threatens successful treatment outcomes. Medication non-adherence is often associated with negative consequences, including symptom exacerbation, more frequent emergency room visits, re-hospitalizations and relapse. Long-acting injectable (LAI) forms of antipsychotics allow for rapid identification of non-adherence, obviate the need for the patient to take the medication on a daily basis and increase adherence to some significant degree. Eli Lilly has developed a long-acting depot formulation of olanzapine, olanzapine pamoate, which has recently been approved by the FDA for the US market, and which will be reviewed here. Olanzapine LAI appears to be an effective antipsychotic at dosages of $210 \mathrm{mg}$ every 2 weeks, $300 \mathrm{mg}$ every 2 weeks and $405 \mathrm{mg}$ every 4 weeks in patients with acute schizophrenia, and at $150 \mathrm{mg}$ every 2 weeks, $300 \mathrm{mg}$ every 2 weeks and at $405 \mathrm{mg}$ every 4 weeks for the maintenance treatment of stable patients. Oral supplementation appears not to be needed, particularly not at the onset of treatment with the LAI as is necessary with risperidone LAI. Its efficacy is in general comparable to the efficacy seen with oral olanzapine at a corresponding dose. The side effect profile is also comparable to the side effects observed with oral olanzapine, including lower rates of extrapyramidal symptoms, prolactin elevation and cardiovascular side effects, but significant metabolic effects. The latter include significant weight gain, lipid abnormalities and glucose dysregulation. While the injection site adverse events are overall mild, the most significant serious adverse event is the post-injection delirium sedation syndrome (PDSS). While rare, this syndrome results from inadvertent intravascular injection of olanzapine LAI and can cause a range of olanzapine overdose-type of symptoms. Olanzapine LAI needs therefore to be administered by trained personnel in settings where a post-injection observation period for at least 3 hours by medical personnel is available. The overall use of olanzapine LAI will probably be limited by the possibility of a PDSS event. Patients who have a history of good response to oral olanzapine and are in need of assured medication administration may present a good indication for its use, provided that the appropriate mental health delivery setting is available.

Keywords: olanzapine, risperidone, schizophrenia

\section{Introduction}

Medication non-adherence in patients with schizophrenia continues to be a significant problem and threatens successful treatment outcomes. ${ }^{1}$ Reported rates of non-adherence to antipsychotics range from $20 \%$ to $69 \%$ with an estimated average rate of about $50 \%{ }^{2,3}$ Medication non-adherence is often associated with negative consequences, including symptom exacerbation, more frequent emergency room visits, re-hospitalizations and relapse. ${ }^{4}$ Among patients who discontinue their medication, $75 \%$ experience significant symptom exacerbation over 1 year compared to $25 \%$ of those who adhere to their 
medication. ${ }^{4}$ In turn, symptom exacerbation can often lead to serious consequences, including dangerous behaviors, worsened prognosis of the disease, antipsychotic treatment resistance and increased healthcare costs. ${ }^{4}$

Medication status is the largest predictor of relapse risk in schizophrenia with continuous maintenance of antipsychotic medication resulting in an approximate $70 \%$ reduction in the risk of relapse. ${ }^{3,4}$

Hence, treatments that can increase medication adherence are critically important in the pharmacological treatment of schizophrenia. Long-acting injectable (LAI) forms of antipsychotics fulfill an important role in this domain of treatment. LAI antipsychotics allow for rapid identification of non-adherence, obviate the need for the patient to take the medication every day and increase adherence to some significant degree. ${ }^{2,3}$

Both typical antipsychotics (fluphenazine and haloperidol decanoate) and 2 atypicals (risperidone LAI and paliperidone palmitate) are available in the US as LAI formulations. Clinicians need a wider range of long-acting depot compounds to choose from within the atypical range of antipsychotics as, at present, only 1 atypical is available as a LAI antipsychotic. Risperidone LAI and paliperidone LAI are closely related to the same molecule.

Eli Lilly has developed a long-acting depot formulation of olanzapine, olanzapine pamoate $\left(\right.$ Zyprexa $^{\circledR}$ Relprevv $\left.^{\mathrm{TM}}\right)$, which has been approved by the Food and Drug Administration (FDA) and introduced in the US in 2010. We will review in the following sections its pharmacology and mode of action, its efficacy and safety profile and its place among the present second-generation LAI formulations.

\section{Methods}

The terms olanzapine pamoate and olanzapine long-acting injectable were systematically searched for on pubmed as well as on www.clinicaltrials.gov up to September 2009. In addition, the FDA website http://www.dfda.gov was queried for any available data on these topics. Finally, the Eli Lilly website was reviewed for any additional reported data on olanzapine pamoate. The following meetings were reviewed for reports on olanzapine LAI: Annual Meeting of the Society of Biological Psychiatry, Washington DC, 2008; Annual Meeting of the American Psychiatric Association, Washington DC, 2008, San Francisco CA, 2009; Annual Meeting of the NCDEU, Phoenix, AZ, 2009. Four peer reviewed studies were found on olanzapine pamoate, ${ }^{5-8}$ 4 entries on www.clinicaltrials.gov ${ }^{9-12}$ and 8 abstracts of poster presentations. ${ }^{13-20}$ It is important to note that until
September 2009 few peer reviewed publications were available on the efficacy and safety of olanzapine pamoate, which somewhat limits this review.

\section{Pharmacology and mode of action}

Olanzapine LAI is the salt of pamoic acid and olanzapine that is suspended in an aqueous solution and is injected into the gluteal muscle. ${ }^{19}$ Once injected in the gluteal muscle, the two components of the salt slowly dissociate into separate molecular compounds, olanzapine and pamoic acid. The rate of dissolution of the salt is slow, allowing for a gradual release of olanzapine into the circulation over 2 to 4 weeks. ${ }^{9}$ Other depot antipsychotic preparations use different mechanisms in order to achieve a slow absorption over time. A number of first-generation LAI antipsychotics possess a terminal alcohol $(-\mathrm{OH})$ group, which allows them to be combined with carboxylic acids by a process of esterification. Long-chain esters show high oil solubility and low water solubility. Long-acting injectable formulations of these drugs contain long-chain drug esters (eg, decanoate) dissolved in a vegetable oil. When injected intramuscularly, the oil forms a depot of drug: the drug ester slowly diffuses into the blood stream and is then rapidly hydrolyzed to release the parent drug. Most second-qeneration antipsychotics lack terminal -OH groups suitable for esterification. Modification of release characteristics is brought about by other means: encapsulating the drug into a biodegradable polymer (risperidone) or injecting a suspension (ie, water-insoluble particulate matter in water) of drug compound (olanzapine). ${ }^{21}$

The optimal time to peak concentration should be short to minimize the need for bridging with oral antipsychotic. Risperidone LAI (Risperdal ${ }^{\circledR}$ Consta $^{\circledR}$; Janssen Pharmaceuticals Ltd) uses microspheres which dissolve gradually and release the active compound into circulation. The absorption is slow and only reaches peak plasma levels after 2 weeks and requires therefore a transition with oral risperidone for 2 weeks initially. The absorption of paliperidone LAI is based on the palmitate ester of paliperidone, which is an aqueous suspension utilizing nanocrystal molecules. This formulation increases its surface area and leads to initial rapid medication release and in a short time to steady state. Active paliperidone plasma levels are detected systemically at day one. The slow rate of dissolution of paliperidone palmitate results in a longer halflife that ranges from 25 to 49 days, allowing a once-monthly intramuscular injection schedule. The first-generation antipsychotics haloperidol and prolixin use ester formations with a decanoate, which dissolve slowly over time. 


\section{Pharmacokinetics}

The doses of olanzapine LAI were chosen based on pharmacokinetic and safety results of phase I studies. ${ }^{9,13,19}$ Specific pharmacokinetic data have been reported in an open label study in 282 stable patients with schizophrenia. ${ }^{3}$ Patients received doses of olanzapine LAI at $100 \mathrm{mg}$, $150 \mathrm{mg}, 160 \mathrm{mg}, 200 \mathrm{mg}$ and $300 \mathrm{mg}$ every 2 weeks, and at $210 \mathrm{mg}, 255 \mathrm{mg}, 300 \mathrm{mg}$ and $405 \mathrm{mg}$ every 4 weeks for 24 weeks. Olanzapine plasma concentrations increased 2 - to 3-fold with multiple dosing reaching eventually steady state after about 3 months of dosing. Olanzapine plasma concentrations were sustained throughout the 2- and 4-week injection intervals. Maximum olanzapine plasma concentrations and area under the concentration vs time curve were proportionate to the LAI dose. Time to peak concentration after the injection was 4 days with a half-life of approximatively 26 days. This is an important pharmacokinetic feature as the time to onset of action after an injection is an important criterion for the clinician. The distribution of olanzapine plasma concentration after olanzapine LAI doses of $210 \mathrm{mg}$ every 2 weeks, $300 \mathrm{mg}$ every 2 weeks and $405 \mathrm{mg}$ every 4 weeks were within the 10th and 90th percentiles of the range of olanzapine plasma concentrations found with the olanzapine oral formulation dosed at $5 \mathrm{mg}$ once daily (10th percentile) and $20 \mathrm{mg}$ once daily (90th percentile). Going from oral to olanzapine LAI, Gulliver et $\mathrm{al}^{19}$ recommend the following conversion strategies: for patients stabilized on $10 \mathrm{mg}$ per day of oral olanzapine the starting olanzapine LAI dose should be $210 \mathrm{mg}$ every 2 weeks or $405 \mathrm{mg}$ every 4 weeks. The patient can then be reduced after 2 months to a lower maintenance dose of $150 \mathrm{mg}$ every 2 weeks or $300 \mathrm{mg}$ every 4 weeks. For patients stabilized on $15 \mathrm{mg}$ of oral olanzapine the starting olanzapine LAI dose should be $300 \mathrm{mg}$ every 2 weeks, which after 2 months can be reduced to $210 \mathrm{mg}$ or $405 \mathrm{mg}$ every 4 weeks. For patients stabilized on $20 \mathrm{mg}$ of oral olanzapine the starting dose of olanzapine LAI should be $300 \mathrm{mg}$ every 2 weeks and should remain at that level, if clinically indicated.

The olanzapine pamoate salt dissolves much more rapidly when it is in contact with a larger amount of blood or plasma, resulting in the release of a large amount of olanzapine over a shorter period of time. Therefore, if olanzapine LAI is accidentally injected into vascular or capillary rich tissue, this property can result in very high olanzapine plasma levels rapidly after the injection with significant clinical acute morbidity. This observation underlies the post-injection delirium/sedation syndrome (PDSS) seen in the context of use of olanzapine LAI. It has been observed in clinical trials and will be reviewed below.

\section{Receptor binding data}

Mamo et $\mathrm{al}^{6}$ reported results on a study examining $\mathrm{D}(2)$ receptor occupancy of olanzapine LAI during a multicenter, open-label study exploring $\mathrm{D}(2)$ receptor occupancy of a fixed dose of olanzapine LAI given every 4 weeks. Patients with schizophrenia or schizoaffective disorder previously stabilized on oral olanzapine were switched to olanzapine LAI $300 \mathrm{mg}$ by intramuscular injection every 4 weeks for 6 months. No within-group significant changes were found in scores of the Brief Psychiatric Rating Scale Total or in the Clinical Global Impressions-Severity of Illness score, although 7 patients received oral olanzapine supplementation during the first four injection cycles. To minimize impact on $\mathrm{D}(2)$ occupancy by the supplemental oral olanzapine, positron emission tomography (PET) scans were not completed during injection cycles that required supplemental oral olanzapine. Mean striatal D(2) receptor occupancy, as measured by [(11) C]-raclopride PET, was $69 \%$ on oral olanzapine (5-20 mg/day) and 50\% (trough) on olanzapine LAI at steady state. Following an initial decline, occupancy returned to $84 \%$ of baseline oral olanzapine occupancy after 6 injections. Over the study period, D(2) receptor occupancy and plasma olanzapine concentrations were significantly correlated $(\mathrm{r}=0.76, P=0.001)$. Olanzapine LAI resulted in mean $\mathrm{D}(2)$ receptor occupancy of approximately $60 \%$ or higher at the end of the 6-month study period, ${ }^{6,22}$ a level consistent with antipsychotic efficacy and also found during treatment with oral olanzapine. However, supplemental oral olanzapine or another dosing strategy may be necessary to maintain adequate therapeutic response during the first few injection cycles.

\section{Efficacy and safety studies Acute treatment}

One large, double-blind, placebo controlled, 8-week study of olanzapine LAI in 404 inpatients with acute schizophrenia patients compared $210 \mathrm{mg}$ every 2 weeks, $300 \mathrm{mg}$ every 2 weeks, $405 \mathrm{mg}$ every 4 weeks and placebo in a 1:1:1:1 randomized design. ${ }^{5}$ All three olanzapine doses separated significantly from placebo on the total Positive and Negative Syndrome Scale (PANSS). The two higher doses separated from placebo on the PANSS at day 3, while all three doses separated at day 7 from placebo. Discontinuation rates were $32 \%$ for the $210 \mathrm{mg}$ dose, $33 \%$ for the $300 \mathrm{mg}$ dose, $28 \%$ for the $405 \mathrm{mg}$ dose and $43 \%$ for the placebo group. The 
mean daily benzodiazepine dose used during the trial was not statistically significantly different in the four treatment groups. There were no in-between group differences in the incidence of use of anticholinergics between the four groups; $8.2 \%$ of the placebo group patients received anticholinergics, while $5.0 \%$ to $12.3 \%$ of the olanzapine LAI group patients received anticholinergics. Sedation and increased appetite were more frequently reported for the $300 \mathrm{mg}$ group than for the placebo group. The three olanzapine groups showed a significantly higher change in mean weight gain compared to placebo (3.2-4.8 vs $0.3 \mathrm{~kg} ; P<0.001$ ), a significantly greater increase in fasting cholesterol values as compared to placebo (5.5-10.4 vs $-7.0 \mathrm{mg} / \mathrm{dL} ; P<0.15)$ and a significantly greater increase in fasting triglycerides in the $210 \mathrm{mg}$ and the $400 \mathrm{mg}$ groups compared to placebo (26.3-30.3 vs $-9.4 \mathrm{mg} / \mathrm{mL} ; P<0.16)$, but not for the $300 \mathrm{mg}$ group $(17.6 \mathrm{mg} / \mathrm{dL} ; P=0.55)$. Few injection site reactions were reported and no patient dropped out of the study because of injection site reaction.

In a more recent post-hoc comparison of these study results with a corresponding set of three studies of oral olanzapine, haloperidol and placebo in similarly acutely ill patients, olanzapine LAI was found to show a comparable magnitude of symptom reduction compared to patients treated with a dose range of $2.5 \mathrm{mg}$ to $15 \mathrm{mg}$ oral olanzapine during 6 weeks of treatment. It appeared that the incidence of parkinsonism was slightly lower for olanzapine LAI with $2.3 \%$ to $6.2 \%$, while it was $7.8 \%$ to $18.5 \%$ for oral olanzapine and $41.8 \%$ to $52.5 \%$ for haloperidol. ${ }^{18}$

\section{Maintenance treatment}

Olanzapine LAI was compared to oral olanzapine for the maintenance of antipsychotic response in stable outpatients with schizophrenia in a 24-week double-blind study. ${ }^{7,14}$ Patients were assigned in a partial randomization design to the following treatment groups: olanzapine LAI $405 \mathrm{mg}$ every 4 weeks, $300 \mathrm{mg}$ every 2 weeks, $150 \mathrm{mg}$ every 2 weeks (total $\mathrm{N}=318)$ or $45 \mathrm{mg} / 4$ weeks $(\mathrm{N}=141)$ or to oral olanzapine $10 \mathrm{mg}, 15 \mathrm{mg}$, or $20 \mathrm{mg} /$ day $(\mathrm{N}=144)$ without a placebo group. The oral olanzapine group assignment was constrained by whichever oral dose patients had been stabilized on before entering the trial for 4 to 8 weeks. Each of the olanzapine LAI doses was statistically superior to the olanzapine LAI $45 \mathrm{mg}$ every 4 weeks dose group based on time to exacerbation. In addition, the three active olanzapine LAI dose groups showed effective maintenance of response as measured by PANSS scores as compared to the $45 \mathrm{mg} / 4$ week group, which showed a statistically significant worsening. Significant separation between the olanzapine LAI dose groups $300 \mathrm{mg}$ every 2 weeks, $405 \mathrm{mg}$ every 4 weeks, $150 \mathrm{mg}$ every 2 weeks groups versus the $45 \mathrm{mg}$ every 4 weeks group in PANSS total scores was also observed starting at 3,2, and 11 weeks, respectively, and was maintained through the end of the study $(P<0.05)$. Similar differences between the three active olanzapine LAI dose groups and the $45 \mathrm{mg}$ every 4 weeks dose group were observed for the Brief Psychiatric Rating Scale total, Clinical Global Impressions-Improvement, and Clinical Global Impressions-Severity (CGI-S) scores. Mean baseline to endpoint change in PANSS total score for the oral olanzapine group was -1.7 , which was not significantly different from that of the highest olanzapine LAI dose group $(300 \mathrm{mg}$ every 2 weeks $=-2.2 ; P=0.606)$, but superior to the other olanzapine LAI doses $(P<0.01)$. The pooled $150 \mathrm{mg}$ and $300 \mathrm{mg}$ every 2 weeks olanzapine LAI groups were non-inferior to their corresponding oral olanzapine dose groups in terms of the non-exacerbation rate over 24 weeks (90\% vs 93\% respectively). Conversely, the three active olanzapine LAI groups (405 mg; $300 \mathrm{mg} ; 150 \mathrm{mg}$ ) showed non-inferiority as compared to the oral olanzapine groups in terms of exacerbation of symptoms.

Treatment related adverse events leading to discontinuation were overall low and comparable over the four olanzapine LAI groups $(3.1 \%, 2.8 \%, 5.0 \%$ and $4.2 \%)$. Clinically significant weight gain from baseline to endpoint was approximatively dose related: $15.2 \%, 20.7 \%, 16.4 \%$ and $8.3 \%$. There were significant increases in total cholesterol and fasting LDL cholesterol in the three active olanzapine LAI groups over the low dose group of $45 \mathrm{mg}$ every 4 weeks, while no significant in-between group differences in change were seen in fasting triglyceride and glucose levels. There was a non-significant increase of shifts from $<150 \mathrm{mg} / \mathrm{dL}$ to high in fasting triglyceride levels seen in the olanzapine LAI $300 \mathrm{mg}$ every 2 weeks group compared to the other three olanzapine LAI groups. No significant differences in treatment emergent extrapyramidal symptoms were reported between any of the olanzapine LAI dose groups and the overall rate of extrapyramidal symptoms was low throughout the trial. Incidence of injection site reactions was low (2.8\%). However, 2 patients treated with olanzapine LAI experienced PDSS following possible inadvertent intravascular injection.

Interim results from another long-term, open-label safety extension study of olanzapine LAI with maximum treatment duration of 190 weeks were recently reported. ${ }^{15}$ Patients were 18 to 75 years of age with schizophrenia or schizoaffective disorder $(\mathrm{N}=931)$ who were followed up after completing 1 of 3 randomized, controlled studies 
of olanzapine LAI, in which patients had been randomly assigned to oral olanzapine, olanzapine LAI, or placebo. During the open-label extension, all patients received flexibly-dosed olanzapine LAI at injection intervals of approximately 2 to 4 weeks. At the time of the report, the rate of study discontinuation was $46.3 \%$. Discontinuation rate at 18 months was $34.3 \%$. The most common reasons for discontinuation were: subject decision (23.4\%), adverse event (6.7\%), and lost to follow-up (5.7\%). Adverse events in $>5 \%$ of patients were increased weight, insomnia, anxiety, somnolence, headache, and nasopharyngitis. Of note were 26 occurrences of PDSS following possible accidental intravascular injection of a portion of the dose; all of the patients fully recovered within 72 hours.

Mean weight change was $+1.88 \mathrm{~kg}$, with $32.1 \%$ of patients experiencing clinically significant weight gain. Percentages of patients who showed an increase from normal to high on fasting glucose, random total cholesterol, or random triglycerides were $5.5 \%, 5.2 \%$, and $14.3 \%$, respectively. Mean CGI-S scores remained stable throughout the open label follow-up of the three studies ( 2.9 at baseline to 2.8 at endpoint).

\section{Switching studies}

Two switching strategies from oral antipsychotics to olanzapine LAI were compared in a recent report: ${ }^{16,17}$ a direct switch from the previous antipsychotic to olanzapine LAI versus taper of previous antipsychotic medication during the first 2 weeks of treatment. Analyses were based on 8-month data from an ongoing 2-year open-label study of olanzapine LAI in outpatients considered at risk for relapse $(\mathrm{N}=264)$ who received olanzapine LAI every 4 weeks with a starting dose of $405 \mathrm{mg}$ and flexible dosing thereafter. Investigators, at their discretion, could either directly switch patients or taper their previous antipsychotic medication during the first 2 weeks of treatment. At the time of study entry, 62 patients were receiving typical antipsychotics, 188 were receiving atypical antipsychotics (76 receiving oral olanzapine), and 34 were not receiving any antipsychotic; a total of 16 were on prior injectable antipsychotic medication. Some patients were taking more than one antipsychotic at baseline. Of a total of 264 patients, 150 (56.8\%) were switched directly and the rest were tapered. The two groups did not significantly differ in discontinuation rates (direct: $29.3 \%$ vs taper: $28.9 \%, P \sim 1.0$ ) and there was no significant difference between the two groups on PANSS total score mean change at any visit up to 8 months (direct: -1.5 , taper: $-3.4, P=0.785$, from a mean baseline of 56.7 [SD $=9.8]$ ). Treatment-emergent adverse events in $\geq 5 \%$ of patients were: increased weight $(10.2 \%)$, insomnia $(8.3 \%)$, anxiety $(6.8 \%)$, somnolence $(6.8 \%)$, and increased appetite $(5.7 \%)$. The switch groups did not significantly differ in mean weight change $(P=0.419)$, with an average weight gain of $2.0 \mathrm{~kg}$, nor did they significantly differ in terms of laboratory analytes or other safety parameters. There did not appear to be clinically significant differences for those who were directly switched to olanzapine LAI versus those who were tapered.

\section{Post-injection syndrome}

A small number of patients have experienced PDSS during the participation in olanzapine LAI trials, which is a serious adverse event. The signs and symptoms include feelings of tiredness, dizziness and fatigue progressing to delirium and or excessive sedation and are probably related to an inadvertent intravascular injection of part or the entire olanzapine LAI dose. In a poster by the manufacturer, all safety data of all completed or ongoing olanzapine LAI trials were pooled up to data lock up to May $312008 .^{20}$ Twenty-nine cases (in 28 patients) of PDSS were identified and reviewed. The per-injection rate was calculated based on a total of $>40,000$ injections as $0.07 \%$ and a per-patient rate of $1.2 \%$. The cumulative risk of experiencing an episode of PDSS after 1 year of treatment was $07 \%$ to $1.2 \%$. The presenting symptoms were consistent with excessive systemic levels of olanzapine LAI, such as sedation, dizziness, confusion, slurred speech, altered gait, weakness, muscle spasms and/or unconsciousness. Two patients experienced significant blood pressure increases, but no other changes in vitals signs were observed. Time to onset varied between 0 to 300 minutes with a mean of 48 minutes. Eighty percent of PDSS events occurred within 1 hour after the injection, $17 \%$ occurred between 1 hour to 3 hours and 1 case occurred after 3 hours. $79 \%$ of patents experiencing a PDSS event were hospitalized and received either only observational care or supportive medical care. Pharmacokinetic data collected at the time of the event revealed that olanzapine plasma concentrations were much higher than expected relative to the post injection concentration previously collected for the same patients. There was no relationship of the PDSS event and any concomitant medications. All patients recovered completely after 3 to 72 hours. PDSS occurred at any injection time point as it was observed from the first injection to the 66th injection. It is interesting to note that PDSS like events have not been reported with other long-acting depot antipsychotics.

The pathophysiology of the PDSS is most likely related to the observation that the olanzapine pamoate salt dissolves much more rapidly when it is in contact with a larger amount 
of blood or plasma, releasing therefore a large amount of olanzapine over a short period of time. Hence, if olanzapine LAI is accidentally injected into vasculature or capillary rich tissue, this property can result in very high olanzapine plasma levels leading to the symptoms of PDSS.

Special precautions need to be taken therefore when using olanzapine LAI. First, proper injection technique needs to used, which includes deep gluteal injection and aspiration of the syringe for 5 seconds in order to verify that no blood is being aspired. In the event of blood being aspired the injection site needs to be changed, the syringe needs to be discarded and a new dose needs to be reconstituted. After the injection the patient needs to be observed for 3 hours at the facility where the injection was administered, and the patient needs to be accompanied home by an informed person. Instructions for the patient need to include information about the symptoms of PDSS, the avoidance of driving or operating machinery. ${ }^{20}$

The overall use of olanzapine LAI will probably be limited by the potential occurrence of a PDSS event. The primary settings where olanzapine LAI will be utilized will be settings where the post-injection observation period can be implemented and easily integrated into the usual service delivery process, such as inpatient settings, day hospitals or day programs where patients can be involved in active group activities and are at the same time under observation by a mental health care provider after the injection.

The Committee for Medicinal Products for Human Use in Europe has recommended approval of olanzapine LAI in September 2008, which was subsequently approved by the European Commission for maintenance treatment of adult patients with schizophrenia sufficiently stabilized during acute treatment with oral olanzapine for the European Community countries. In contrast, the FDA has delayed a decision on approval as recently as September 2009. The Agency has requested that Eli Lilly and Company prepare a proposed risk evaluation and mitigation strategy. In February 2009 the FDA had issued a not-approvable letter, saying it required additional information about the risk and cause of excessive sedation observed in some patients. Finally, olanzapine LAI was approved by the FDA with a black box warning in 2010. The specific requirements are that olanzapine LAI must be administered in a registered healthcare facility with ready access to emergency response services. After each injection, patients must be observed at the healthcare facility by a healthcare professional for at least 3 hours. Because of this risk, olanzapine LAI is available only through a restricted distribution program called ZYPREXA
RELPREVV Patient Care Program and requires prescriber, healthcare facility, patient, and pharmacy training.

\section{Comparison with other long-acting antipsychotics}

No data have yet been published on head-to-head randomized trials comparing olanzapine LAI with other antipsychotic depot preparations. There is, however, a virtual comparison examining completion rates of olanzapine LAI studies and of studies with risperidone (RLAI) in LAI formulations. Completion rates were used as a proxy of overall efficacy during the 12-month open-label treatment of patients with schizophrenia. ${ }^{8}$ Two comparisons of 12-month completion rates were made: (1) between the two OLAI and RLAI studies which had the most similar study design and patient characteristics; and (2) in all relevant OLAI and RLAI studies identified. For the first comparison, 12-month completion rates for olanzapine LAI and RLAI were $81.5 \%$ and $52.0 \%$ $(P<0.001)$, respectively, for the subgroup of the studies, which included the most similar populations with regard to baseline treatment and patient characteristics. For the second comparison, 12-month completion rates were $72.9 \%$ and 58.0\% $(P<0.001)$, respectively, for all patients included in Study HGKB and a pooled analysis of all relevant RLAI studies. No individual RLAI study had a 12-month completion rate greater than that seen for OLAI. However, these results have to be tempered given the potential imbalance between populations across the selected studies.

\section{Conclusions}

This review is somewhat limited by the fact that few peer reviewed publications are as yet available on the efficacy and safety of olanzapine pamoate. Overall, olanzapine LAI appears to be an effective antipsychotic at dosages of $210 \mathrm{mg}$ every 2 weeks, $300 \mathrm{mg}$ every 2 weeks and $405 \mathrm{mg}$ every 4 weeks in patients with acute schizophrenia, and at $150 \mathrm{mg}$ every 2 weeks, $300 \mathrm{mg}$ every 2 weeks and at $405 \mathrm{mg}$ every 4 weeks for the maintenance treatment of stable patients with schizophrenia. Its efficacy is in general comparable to the efficacy seen with oral olanzapine at a corresponding dose. The side effect profile is also comparable to the side effects observed with oral olanzapine, including lower rates of extrapyramidal symptoms, prolactin elevation and cardiovascular side effects, but significant metabolic effects. The latter ones include significant weight gain, lipid abnormalities (increase in cholesterol, low-density lipoprotein cholesteraol levels and triglycerides) and glucose dysregulation. While the injection site adverse events are overall mild, the most significant serious 
adverse event is the PDSS. While rare, this syndrome results from inadvertent intravascular injection of olanzapine LAI and can cause a range of olanzapine overdose-type of symptoms. Olanzapine LAI needs therefore to be administered by trained personnel in settings where a post-injection observation period for at least 3 hours by medical personnel is available. The overall use of olanzapine LAI will probably be limited by the possibility of a PDSS event. Patients who have a history of good response to oral olanzapine and are in need of assured medication administration may present a good indication for its use, provided that the appropriate mental health delivery setting is available.

\section{Disclosures}

JP Lindenmayer, MD has received research grants from Eli Lilly, Pfizer, Organon, Otsuka, Dainippon-Sumitomo, Bristol-Myers Squibb and Johnson and Johnson. He is a member of Advisory Boards of Eli Lilly and Johnson and Johnson.

\section{References}

1. Keith SJ, Kane JM. Partial compliance and patient consequences in schizophrenia: our patients can do better. $J$ Clin Psychiatry. 2003;64(11):1308-1315.

2. Kane JM, Leucht S, Carpenter D, et al. The expert consensus guideline series. Optimizing pharmacologic treatment of psychotic disorders. Introduction: methods, commentary, and summary. J Clin Psychiatry. 2003;64(Suppl 12):S5-S19.

3. Nasrallah HA. The case for long-acting antipsychotic agents in the postCATIE era. Acta Psychiatr Scand. 2007;115(4):260-267.

4. Kane JM. Strategies for improving compliance in treatment of schizophrenia by using a long-acting formulation of an antipsychotic: clinical studies. J Clin Psychiatry. 2003;64(Suppl 16):S34-S40.

5. Lauriello J, Lambert T, Andersen S, et al. An 8-week, double-blind, randomized, placebo-controlled study of olanzapine long-acting injection in acutely ill patients with schizophrenia. J Clin Psychiatry. 2008;69(5):790-799.

6. Mamo D, Kapur S, Keshavan M, et al. D2 receptor occupancy of olanzapine pamoate depot using positron emission tomography: an open-label study in patients with schizophrenia. Neuropsychopharmacology. 2008;33(2):298-304.

7. Detke H, McDonnell D, Kane J, et al. Olanzapine long-acting injection for the maintenance treatment of schizophrenia: a 24-week, randomized, double-blind trial. Schizophr Res. 2008;102(Suppl 2):262-263.

8. Ascher-Svanum H, Montgmery W, Brnabic A, et al. Treatment completion rates of olanzapine and risperidone in long acting injectable formulations for the treatment of schizophrenia: a comparison of single arm open-label studies. Schizophr Res. 2008;(Suppl 2):278.
9. Eli Lilly and Company; Pharmacokinetic Characterization of Intramuscular Olanzapine Depot (Clinical Trails ID: NCT00094640; F1D-EW-LOBS). http://clinicaltrials.gov/ct2/show/NCT00094640? term $=$ NCT00094640\&rank=1.

10. Eli Lilly and Company; Comparison of Intramuscular Olanzapine Depot With Placebo in the Treatment of Patients With Schizophrenia (Clinical Trails ID: NCT00088478, F1D-MC-HGJZ). http://clinicaltrials.gov/ct2/ show/NCT00088478?term=NCT00088478\&rank=1.

11. Eli Lilly and Company; Open-Label Study of Intramuscular Olanzapine Depot in Patients With Schizophrenia or Schizoaffective Disorder (Clinical Trails ID: NCT00088465). http://clinicaltrials.gov/ct2/show/ NCT00088465?term $=$ NCT00088465\&rank $=1$.

12. Eli Lilly and Company. Olanzapine Pamoate Depot Versus Oral Olanzapine on Treatment Outcomes in Outpatients With Schizophrenia. ClinicalTrials ID: NCT00320489. http://clinicaltrials.gov/ct2/show/ NCT00320489?term=NCT00320489\&rank $=1$.

13. Kuntz H, Bergstroom R, MsDonnell D, et al. Pharmocokinetics (PK) of multiple doses of olanzapine long acting injection (OLAI), an intramuscular (IM) depot formulation of olanzapine (OLZ), in stabilized patients with schizophrenia. Biol Psychiatry. 2008;63:288S.

14. Detke H, McDonnell D, Kane J, et al. Efficacy and safety of olanzapine long-acting injection for maintenance treatment of schizophrenia. Eur Neuropsychopharmacol. 2008;18(Supp1 4):S393-S394.

15. McDonnell D. Long-term open-label safety of olanzapine long-acting injection: 190-week interim results. American Psychiatric Association. 162nd Annual Meeting 2009:Abstract NRI-060:25.

16. Detke H, Zhao F, Andersen S, et al. Comparison of olanzapine longacting injection switching methods: an 8-month analysis of patients with schizophrenia of risk of relapse. American Psychiatric Association. 162nd Annual Meeting 2009:Abstract NRI-023:9.

17. Detke H, McDonnell D, Andersen S, et al. Olanzapine long-acting injection in patients with schizophrenia at risk of relapse: 12-week switching data. Eur Neuropsychopharmacol. 2008;18(Suppl 14):S435.

18. Zhao F, Detke H, Carlson J, et al. Acute Efficacy of olanzapine long-acting injection, oral olanzapine, and haloperidol in patients with schizophrenia: cross-study comparison. American Psychiatric Association. 162nd Annual Meeting 2009:Abstract NRI-099:40.

19. Gulliver A, Detke H, McDonnell DP, et al. Olanzapine Long acting injection: Pharamcokinetics and dose correspondence data relative to oral olanzapine. Int J Neuropsychopharamcol. 2008;11(Suppl 1):152-153.

20. McDonnel D, Sorsaburu S, Brunner E, Detke H, et al. Postinjection delirium/sedation syndrome observed with olanzapine long acting injection: Review of the first 25 events. Eur Neuropsychopharmacol. 2008;18(Suppl 4):S437-S438.

21. Taylor D. Psychopharmacology and adverse effects of antipsychotic long-acting injections: a review. Br J Psychiatry. 2009;195:S13-S19.

22. Uchida H, Mamo DC, Kapur S, Labelle A, Shammi C, Mannaert EJL, et al. Monthly administration of long-acting injectable risperidone and striatal dopamine D2 receptor occupancy for the management of schizophrenia. J Clin Psychiatry. 2008;69(8):1281-1286.
Neuropsychiatric Disease and Treatment

\section{Publish your work in this journal}

Neuropsychiatric Disease and Treatment is an international, peerreviewed journal of clinical therapeutics and pharmacology focusing on concise rapid reporting of clinical or pre-clinical studies on a range of neuropsychiatric and neurological disorders. This journal is indexed on PubMed Central, the 'PsycINFO' database and CAS, and is the official

\section{Dovepress}

journal of The International Neuropsychiatric Association (INA). The manuscript management system is completely online and includes a very quick and fair peer-review system, which is all easy to use. Visit http://www.dovepress.com/testimonials.php to read real quotes from published authors. 